Full Paper: The copolyperoxides of various compositions of indene with methyl acrylate, ethyl acrylate and butyl acrylate have been synthesized by the free-radicalinitiated oxidative copolymerization. The compositions of copolyperoxide obtained from ${ }^{1} \mathrm{H}$ and ${ }^{13} \mathrm{C}$ NMR spectra have been used to determine the reactivity ratios of the monomers. The copolyperoxides contain a greater proportion of the indene units in random placement. The NMR studies have shown irregularities in the copolyperoxide chain due to the cleavage reactions of the propagating peroxide radical. The thermal analysis by differential scanning calorimetry suggests alternating peroxide units in the copolyperoxide chain. From the activation energy for the thermal degradation, it was inferred that degradation occurs via the dissociation of the peroxide $(\mathrm{O}-\mathrm{O})$ bonds of the copolyperoxide chain. The flexibility of the polyperoxides in terms of glass transition temperature $\left(T_{\mathrm{g}}\right)$ has also been examined.

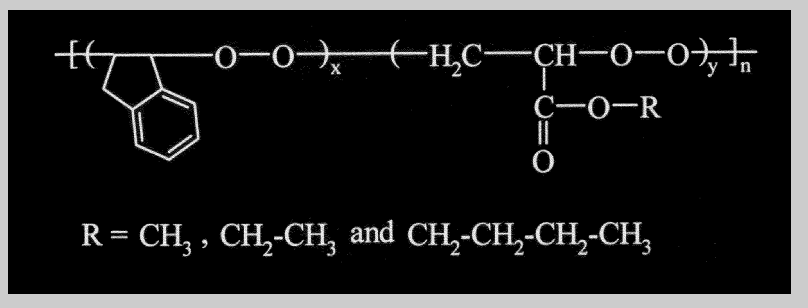

\title{
Determination of the Reactivity Ratios for the Oxidative Copolymerizations of Indene with Methyl, Ethyl and Butyl Acrylates
}

\author{
Priyadarsi De, D. N. Sathyanarayana* \\ Department of Inorganic and Physical Chemistry, Indian Institute of Science, Bangalore 560012, India \\ Fax: +91 80 3600683; E-mail: dns@ipc.iisc.ernet.in
}

Keywords: chain irregularities; copolymerization; differential scanning calorimetry (DSC); exothermic degradation; reactivity ratio

\section{Introduction}

Copolymerization and blending are commonly used to obtain polymers with desired properties. Since polymers are generally immiscible due to adverse entropy effects, copolymerization offers a convenient method of incorporating different monomers in the polymer chain. The difficulties associated with the synthesis of copolymers are the wide variation in the reactivity ratios of the monomers. Polymerization of vinyl monomers in the presence of high pressures of oxygen, called oxidative polymerization yields alternating copolymers with oxygen, known as polyperoxides. ${ }^{[1,2]}$ They find importance as polymeric fuels, ${ }^{[3]}$ thermal, ${ }^{[4,5]}$ photo $^{[6]}$ and base catalyzed initiators ${ }^{[7]}$ for vinyl monomers to synthesize homopolymers, block copolymers and novel comb polymers and curators in coating and moulding applications. ${ }^{[8]}$

Polyperoxides have gained considerable interest over the past several years because of their unique highly exothermic pyrolysis behavior, ${ }^{[9]}$ in contrast to other common polymers which generally degrade endothermically. To improve the quality of polymeric coatings and adhesives, the use of mixtures of monomers and cross-linking agents has attracted attention. ${ }^{[10]}$ In this context, it is desirable to understand the mechanism of copolymerization of a mixture of monomers in the presence of oxygen.

The term oxidative copolymerization ${ }^{[2,11]}$ is applied when two monomers $\left(R_{1}\right.$ and $\left.R_{2}\right)$ reacting in the presence of oxygen at high pressures, produce copolymers of the type $\left.+\left(\mathrm{R}_{1}-\mathrm{O}-\mathrm{O}\right)_{\mathrm{X}}\left(\mathrm{R}_{2}-\mathrm{O}-\mathrm{O}\right)_{\mathrm{Y}}\right\}_{\mathrm{n}}$. Compared to simple vinyl polyperoxides obtained from a vinyl monomer and oxygen, those obtained by the copolymerization of a mixture of vinyl monomers in the presence of oxygen have been less studied. ${ }^{[2]}$ In an earlier work on the oxidative copolymerization, relative rates of oxidation of different monomers and their reactivity towards a peroxide radical was investigated. ${ }^{[12,13]}$ The oxidation of two monomers can be considered as a special case of terpolymerization, when the monomers do not homopolymerize. The uniqueness of this system is that it approximates to a binary copolymerization in terms of $-\mathrm{RO}_{2}^{\circ}$ radicals. ${ }^{[14]}$

Among the monomers, indene forms the most stable solid polyperoxide. ${ }^{[15]}$ The polymeric peroxides of methyl acrylate, ethyl acrylate and butyl acrylate are extremely unstable and difficult to isolate from the reaction mix- 
ture. ${ }^{[12]}$ On oxidation, these monomers usually yield low molecular weight oxidation products rather than the polyperoxides. In the present work, the oxidative copolymerization of three systems, indene-methyl acrylate, indeneethyl acrylate and indene-butyl acrylate was investigated. However, indene does not form normal copolymers with these monomers by free radical methods. Unlike some other vinyl monomers, indene does not homopolymerize, even at low oxygen concentration. ${ }^{[16]}$ The objective of the present study is to determine the reactivity ratios of the monomers forming the copolyperoxides by nuclear magnetic resonance spectroscopy. Other physical techniques namely FTIR and DSC have used to further characterize the polyperoxides.

\section{Experimental Part}

\section{Materials}

Methyl acrylate (MA), ethyl acrylate (EA) and butyl acrylate (BA) (Rolex Laboratory Reagents, India) were freed from inhibitor by washing them first with $5 \% \mathrm{NaOH}$ and then repeatedly with water. After drying over anhydrous $\mathrm{Na}_{2} \mathrm{SO}_{4}$, they were distilled under reduced pressure. Indene (I) (Aldrich, USA) was shaken with $6 \mathrm{M} \mathrm{HCl}$ for $2 \mathrm{~h}$, then refluxed with $40 \% \mathrm{NaOH}$ for $2 \mathrm{~h}$ and fractionally distilled. The distillate was passed through a column of activated silica gel. It was finally distilled under reduced pressure. 2,2'-Azoisobutyronitrile (AIBN) (Koch Light Laboratories, England) was recrystallized twice from methanol. High purity oxygen (Bhoruka gases, 99.9\%) was used. Reagent grade solvents (Ranbaxy, India) namely petroleum ether, benzene, $\mathrm{CH}_{2} \mathrm{Cl}_{2}$, etc. were purified by standard procedures.

\section{Synthesis of Copolyperoxides}

Copolyperoxides were prepared using benzene solutions of the monomers and AIBN as initiator $\left(0.01 \mathrm{~mol} \cdot \mathrm{L}^{-1}\right)$ in a 300 $\mathrm{mL}$ Parr reactor (Parr Instruments Co., USA) equipped with a digital pressure transducer, temperature controller and a mechanical stirrer. The polymerizations were carried out at $50 \pm 0.5^{\circ} \mathrm{C}$ for $6-84 \mathrm{~h}$ under an oxygen pressure of $100 \mathrm{psi}$. The feed ratio was varied to get terpolymers of different compositions. Conversion, based on both acrylate and indene monomers, was kept below $15 \%$. The polymers were isolated from the reaction mixture using petroleum ether as a non-solvent and purified by repeated precipitation from benzene solution followed by the removal of the solvent by vacuum drying. Details regarding the synthesis and identification of the copolyperoxides are given in Table 1.

\section{Measurements}

The FTIR spectrum was recorded on a Bruker Equinox 55 FTIR spectrometer. The thermal analysis was carried out using a Perkin-Elmer DSC-2C differential scanning calorimeter (DSC) under nitrogen atmosphere at heating rates of 5, $10,20,40$ and $80^{\circ} \mathrm{C} / \mathrm{min}$ with sample size of $1-5 \mathrm{mg}$. The molecular weight of the polymers were obtained using a Waters HPLC/GPC instrument (refractive index detector) with THF as a mobile phase at a flow rate of $1.0 \mathrm{~mL} \cdot \mathrm{min}^{-1}$ at $30^{\circ} \mathrm{C}$ using polystyrene standards.

The ${ }^{1} \mathrm{H}$ and ${ }^{13} \mathrm{C}$ NMR spectra were recorded at ambient temperature on a Bruker ACF $200 \mathrm{MHz}$ spectrometer in $\mathrm{CDCl}_{3}$ and $\mathrm{CH}_{2} \mathrm{Cl}_{2}\left(\mathrm{D}_{2} \mathrm{O}\right.$ external lock). The chemical shifts are measured with reference to tetramethylsilane (TMS). The ${ }^{13} \mathrm{C}$ NMR spectra were obtained under inverse gated decoupling with $15 \mathrm{~s}$ delay between the pulses. A line broadening of $2 \mathrm{~Hz}$ was applied before Fourier transformation in order to enhance the signal-to-noise ratio.

Table 1. Experimental results for the oxidative copolymerization of indene with methyl acrylate, ethyl acrylate and butyl acrylate, initiated by AIBN at $50^{\circ} \mathrm{C}$.

\begin{tabular}{|c|c|c|c|c|c|c|c|}
\hline \multirow[t]{3}{*}{ Polymer } & \multirow{3}{*}{$\frac{\text { Reaction time }}{\mathrm{h}}$} & \multirow{3}{*}{$\frac{\text { Yield }}{\%}$} & \multicolumn{3}{|c|}{ Mole fraction of indene } & \multirow[t]{3}{*}{$\bar{M}_{\mathrm{n}}$} & \multirow[t]{3}{*}{$P D$} \\
\hline & & & \multirow[t]{2}{*}{ Feed $[\mathrm{I}]$} & \multicolumn{2}{|c|}{ Copolyperoxide } & & \\
\hline & & & & ${ }^{1} \mathrm{H}$ NMR & ${ }^{13} \mathrm{C}$ NMR & & \\
\hline \multicolumn{8}{|c|}{ Indene/methyl acrylate } \\
\hline PIP & 6 & 12.8 & 1.000 & 1.000 & 1.000 & 3240 & 1.15 \\
\hline IMA1 & 8 & 11.7 & 0.425 & 0.929 & 0.946 & 3280 & 1.45 \\
\hline IMA2 & 12 & 12.7 & 0.240 & 0.878 & 0.883 & 3140 & 1.15 \\
\hline IMA3 & 24 & 11.2 & 0.076 & 0.658 & 0.674 & 3020 & 1.72 \\
\hline IMA4 & 48 & 9.3 & 0.035 & 0.301 & 0.324 & 2700 & 1.56 \\
\hline PMAP & 80 & 7.0 & 0.000 & 0.000 & 0.000 & & \\
\hline \multicolumn{8}{|c|}{ Indene/ethyl acrylate } \\
\hline IEA1 & 8 & 11.0 & 0.451 & & 0.931 & 2660 & 1.39 \\
\hline IEA2 & 14 & 12.8 & 0.269 & & 0.840 & 3220 & 1.24 \\
\hline IEA3 & 30 & 11.1 & 0.087 & & 0.605 & 3020 & 1.13 \\
\hline IEA4 & 65 & 7.7 & 0.044 & & 0.324 & 2400 & 1.64 \\
\hline \multicolumn{8}{|c|}{ Indence/butyl acrylate } \\
\hline IBA1 & 12 & 10.5 & 0.291 & & 0.887 & 3000 & 1.23 \\
\hline IBA2 & 48 & 12.2 & 0.120 & & 0.617 & 2960 & 1.53 \\
\hline IBA3 & 72 & 9.2 & 0.058 & & 0.340 & 2130 & 1.21 \\
\hline IBA4 & 84 & 5.0 & 0.035 & & 0.202 & 1990 & 1.31 \\
\hline
\end{tabular}




\section{Results and Discussion}

\section{Molecular Weight}

Copolymers obtained are either yellowish sticky mass or solid material depending on the ratio of indene to vinyl monomer. The number-average molecular weight $\left(\bar{M}_{\mathrm{n}}\right)$ and polydispersity index $(P D)$ of the polymers are given in Table 1. The polymers have low molecular weight since this category of polymers undergo facile degradation during the oxygen copolymerization, generating chain transfer agents such as aldehydes, which react with macro-growing radicals, resulting in low molecular weight. ${ }^{[17]}$ The copolyperoxides should be stored in dark and in a refrigerator to minimize degradation.

\section{FTIR Spectra}

The FTIR spectra of the neat films of poly(indene peroxide) (PIP), IMA3 and poly(methyl acrylate peroxide) (PMAP) are shown in Figure 1. The spectra are all similar except for the variation in the intensities of the different peaks. The strong band observed near $1025 \mathrm{~cm}^{-1}$ in the spectra is assigned to the peroxide $(-\mathrm{O}-\mathrm{O}-$ ) bond stretching mode. The very intense band at $1740 \mathrm{~cm}^{-1}$ is assigned to the carbonyl groups of MA units and its intensity increases with increase in the MA content of the copolyperoxide. Other carbonyl groups present as end groups also show infrared absorption in this region and hence they are indistinguishable.

The broad absorption in the region $3400-3500 \mathrm{~cm}^{-1}$ is due to the hydroxyl and hydroperoxide end groups. The formation of these end groups via various chain transfer mechanisms has been reported in the literature. ${ }^{[18,19]}$ Similar observation has also been made for I-EA or I-BA series of copolyperoxides.

\section{Copolyperoxide Compositions}

Typical ${ }^{1} \mathrm{H}$ NMR spectra of IMA3 and IMA4 along with those of the homopolyperoxides PIP and PMAP are displayed in Figure 2. The compositions of the copolyperoxides were determined from their ${ }^{1} \mathrm{H}$ and ${ }^{13} \mathrm{C}$ NMR spectra. The NMR spectra have revealed that the monomers do not homopolymerize under high pressures of oxygen employed here. The main chain $\mathrm{CH}_{2}$ and $\mathrm{CH}$ protons show downfield shift for all the polymers due to the two electronegative oxygen atoms to which they are directly bonded. The ${ }^{1} \mathrm{H}$ NMR spectrum of PIP exhibits signals at $7.0-7.5,5.5-5.8$ and $4.6-5.2 \mathrm{ppm}$ corresponding to the aromatic protons, methine protons, one bonded to the aromatic ring and the other to the methylene group, respectively. ${ }^{[15]}$ The two peaks at 2.8 and $3.2 \mathrm{ppm}$ are attributed to the methylene group of indene. ${ }^{[15]}$ In the ${ }^{1} \mathrm{H}$ NMR spectrum of PMAP, the signals at $3.79,4.38$ and $5.22 \mathrm{ppm}$ may be assigned to the methyl, methylene and methine

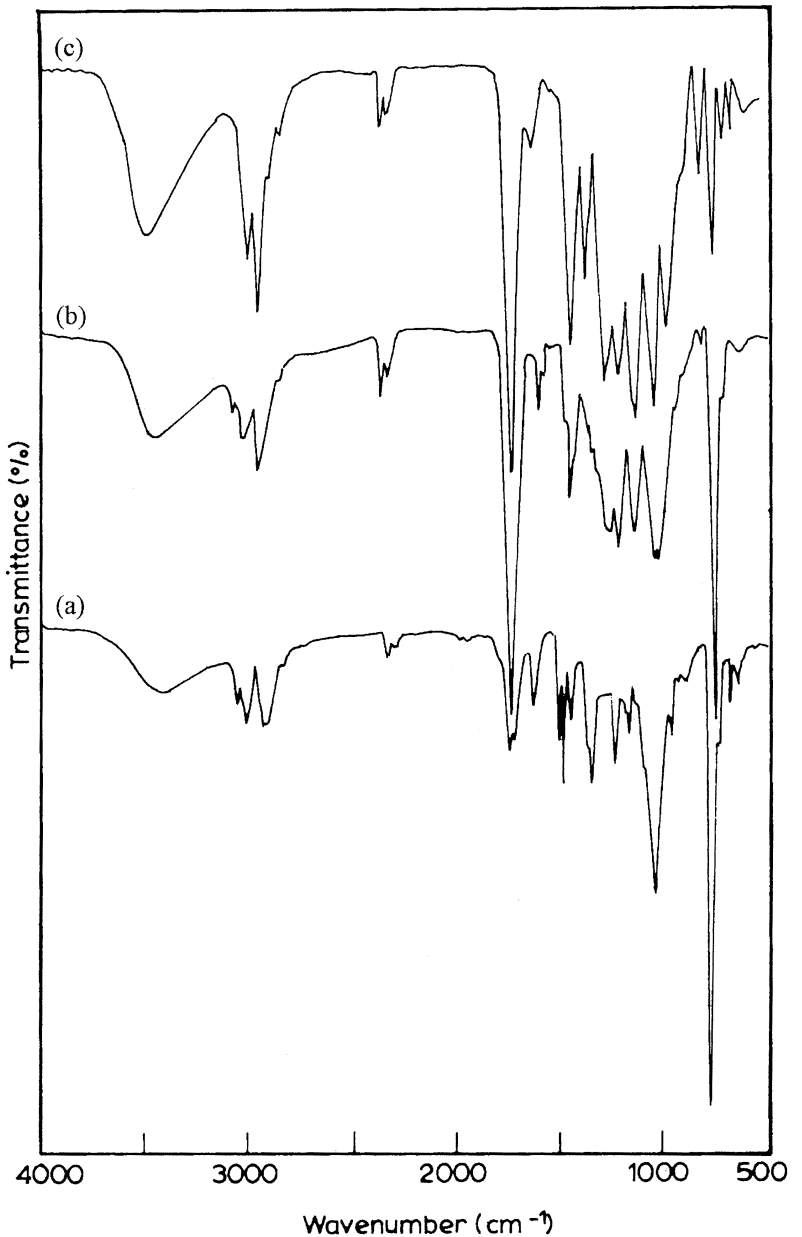

Figure 1. FTIR spectra of (a) PIP, (b) IMA3 and (c) PMAP.

protons, respectively. The copolyperoxide compositions given in Table 1 were obtained from the ratio of the peak intensities of the aromatic protons of the indene units to the methyl protons of MA units.

Similar NMR spectra were observed for indene units in I-EA or I-BA copolyperoxides as described earlier. However EA incorporated into the rigid PIP backbone shows signals at $1.26 \mathrm{ppm}$ for the methyl and in the region $3.88-5.20 \mathrm{ppm}$ for methylene and methine protons. The presence of BA units in the copolymer with indene was confirmed from ${ }^{1} \mathrm{H}$ NMR spectra for this series (Figure 3 ). For these copolyperoxides, we could not estimate the compositions of copolyperoxides from the ${ }^{1} \mathrm{H}$ NMR spectra. Poly(ethyl acrylate peroxide) (PEAP) and poly(butyl acrylate peroxide) (PBAP) could not be prepared due to their extreme instability and very low molecular weight rendering it difficult to precipitate them from the reaction mixture.

However, the ${ }^{13} \mathrm{C}$ NMR spectra recorded under inverse gated decoupling permits the determination of the copolyperoxide compositions. Figure 4 depicts the ${ }^{13} \mathrm{C}$ NMR spectra of the copolyperoxides IMA3, IMA4 and the homopolyperoxides. Considerable downfield shift of the 

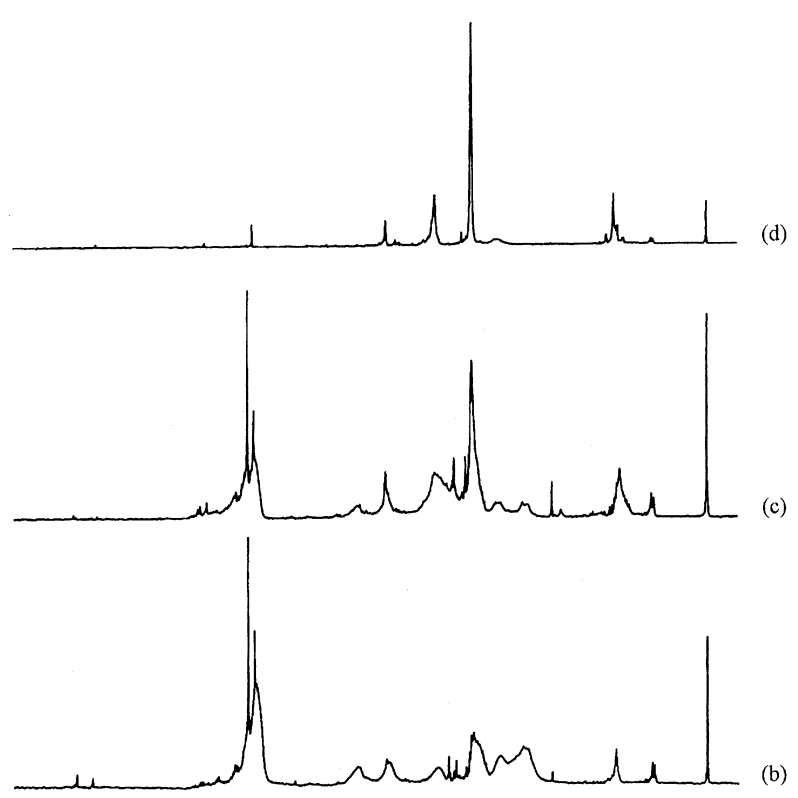

(b)

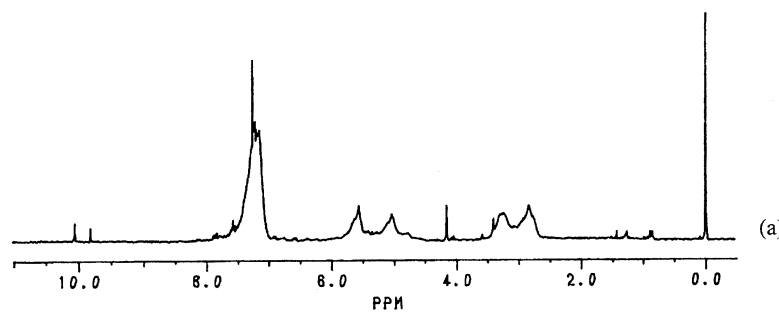

Figure 2. $\quad{ }^{1} \mathrm{H}$ NMR spectra of (a) PIP, (b) IMA3, (c) IMA4 and (d) PMAP in $\mathrm{CDCl}_{3}$.
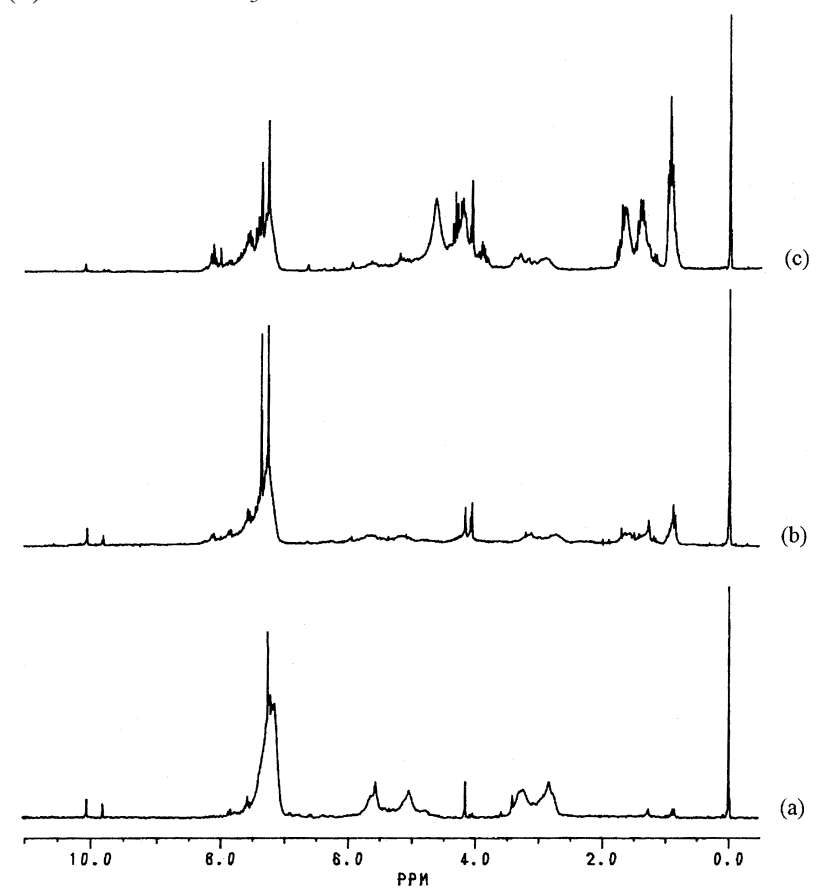

Figure 3. $\quad{ }^{1} \mathrm{H}$ NMR spectra of (a) PIP, (b) IBA3 and (c) IBA4 in $\mathrm{CDCl}_{3}$.

methine and methylene carbons is observed due to the two highly electronegative oxygen atoms to which they are directly bonded. The ${ }^{13} \mathrm{C}$ NMR spectrum of PIP exhi-

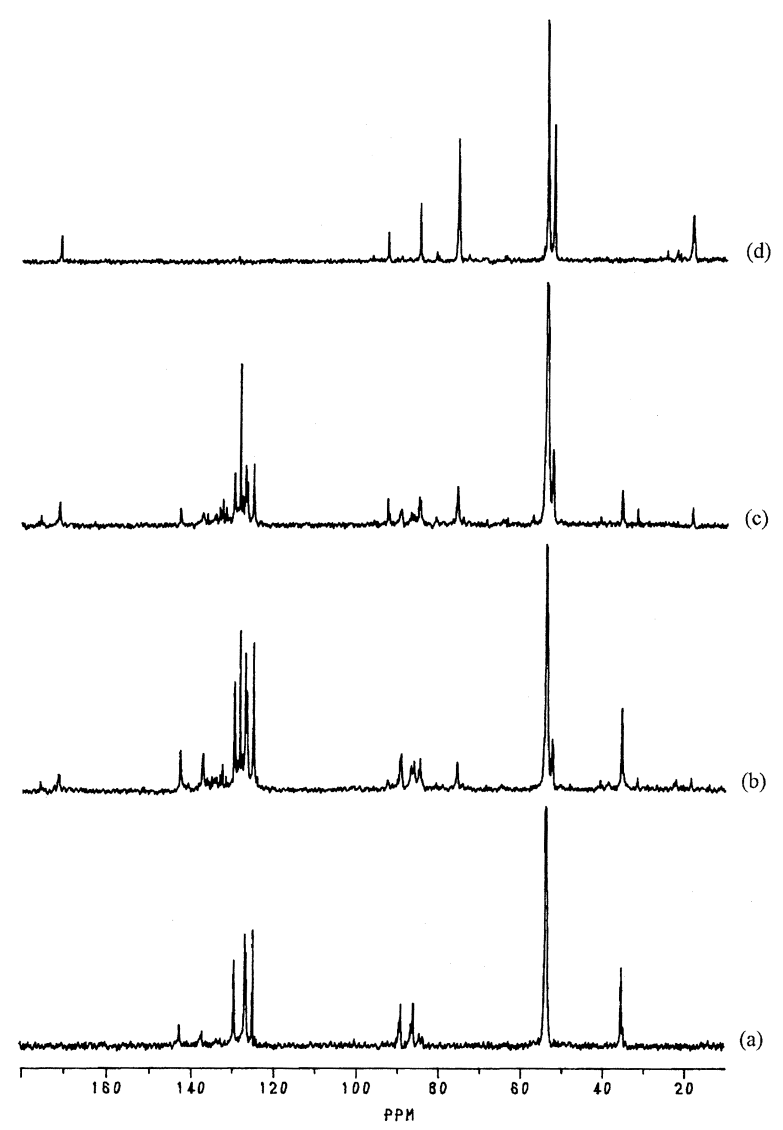

Figure 4. ${ }^{13} \mathrm{C}$ NMR spectra of (a) PIP, (b) IMA3, (c) IMA4 and (d) PMAP in $\mathrm{CH}_{2} \mathrm{Cl}_{2}$.

bits signals at 125-129.6 (aromatic carbons), 137.3 and 142.8 (two aromatic ipso carbons), 89.1 (methine carbon attached to aromatic ring), 86.0 (methine carbon adjacent to the methylene group) and 35.5 (methylene carbon) ppm, respectively. In the PMAP spectrum signals at 52.2, $75.7,84.8$ and $170.9 \mathrm{ppm}$ are attributed as due to the methyl, methylene, methine and carbonyl carbons. The ratio of the intensities between the two types of $\mathrm{CH}_{2}$ carbons yields directly the compositions of copolyperoxide. The ${ }^{13} \mathrm{C}$ NMR spectra provide evidence for the presence of EA and BA units in the copolyperoxides. The ratio of the intensities of the two methylene carbons, $\mathrm{CH}_{2}$ of indene units to the $\mathrm{CH}_{2}$ of the main chain of EA/BA gives a direct measure of the composition of copolyperoxides. The results are listed in Table 1. From the above discussion, the general formula of the copolyperoxide could be represented as:

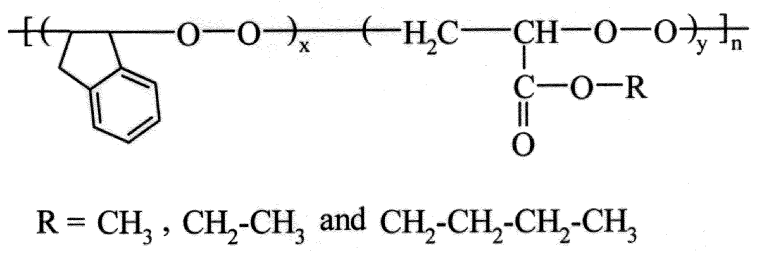

The copolyperoxides exhibit two very weak peaks in the region 9.75-10.2 ppm in the ${ }^{1} \mathrm{H}$ NMR spectra due to 
the two types of $\mathrm{O}=\mathrm{CH}-$ groups present at chain ends. There is a peak at $92.4 \mathrm{ppm}$ in the ${ }^{13} \mathrm{C}$ NMR spectra, which may be attributed to the existence of methylene groups with neighboring peroxy $\left(-\mathrm{O}-\mathrm{CH}_{2}-\mathrm{O}-\right)$ groups in the chain. The presence of $\left(-\mathrm{O}-\mathrm{CH}_{2}-\mathrm{O}-\right)$ moiety in the polyperoxide chain has been reported for the oxidation of $\alpha$-methylstyrene at very low oxygen pressure. ${ }^{[20]}$ It indicates that the cleavage reactions occur to a considerable extent during the oxidative polymerization. This peak is absent in the ${ }^{13} \mathrm{C}$ NMR spectrum of PIP demonstrating that the origin of this peak is from the vinyl monomer in the copolymer. The intensity of this peak increases with the amount of vinyl monomer in polymer.

\section{Reactivity Ratios}

While deriving the equation for the oxidation of two monomers, oxygen is considered as a third monomer. Under the conditions employed here, such as high partial pressure of oxygen, the monomers do not homopolymerize. ${ }^{[2]}$ Further, the propagation reactions involving the addition of one or the other monomer to a second monomer radical is assumed to be negligible. This assumption is supported by the absence of peaks corresponding to IMA, I-EA or I-BA in the ${ }^{1} \mathrm{H}$ and ${ }^{13} \mathrm{C}$ NMR spectra of the copolyperoxides. In the oxidative polymerization, the monomer radical $\left(\mathrm{R}^{\bullet}\right)$ has a higher reactivity with oxygen compared to that of $-\mathrm{RO}_{2}^{*}$ with $\mathrm{R}^{\left[{ }^{[1]}\right.}$ Since in the oxidation of co-monomers, the copolymer composition is not proportional to the feed composition, a penultimate effect could be expected. ${ }^{[21]}$ Therefore, assuming a penultimate effect, the important rate determining propagation steps in the oxidative copolymerization of I with MA may be written as follows: ${ }^{[22]}$

Rate constants

$$
\begin{array}{lll}
-\mathrm{IO}_{2}^{\bullet}+\mathrm{I} & \rightarrow-\mathrm{IO}_{2} \mathrm{I}^{\bullet} & k_{\mathrm{IOI}} \\
-\mathrm{IO}_{2}^{\bullet}+\mathrm{MA} & \rightarrow-\mathrm{IO}_{2} \mathrm{MA}^{\bullet} & k_{\mathrm{IOMA}} \\
-\mathrm{MAO}_{2}^{\bullet} \mathrm{I} & \rightarrow-\mathrm{MAO}_{2} \mathrm{I}^{\bullet} & k_{\mathrm{MAOI}} \\
-\mathrm{MAO}_{2}^{*}+\mathrm{MA} & \rightarrow-\mathrm{MAO}_{2} \mathrm{MA}^{\bullet} & k_{\mathrm{MAOMA}}
\end{array}
$$

Applying the steady state approximation separately for the reactive species, $-\mathrm{IO}_{2}^{*}$ and $-\mathrm{MAO}_{2}^{*}$, the copolymer composition could be expressed in terms of the feed composition and the reactivity ratios,

$\frac{\mathrm{d}[\mathrm{I}]}{\mathrm{d}[\mathrm{MA}]}=\frac{[\mathrm{I}]}{[\mathrm{MA}]} \frac{\left(r_{\mathrm{I}}[\mathrm{I}]+[\mathrm{MA}]\right)}{\left([\mathrm{I}]+r_{\mathrm{M}}[\mathrm{MA}]\right)}$

where $d[I] / d[M A]$ represents the ratio of $I$ to $M A$ in the copolyperoxide, $[\mathrm{I}] /[\mathrm{MA}]$ is the corresponding feed ratios, and $r_{\mathrm{I}}$ and $r_{\mathrm{M}}$ are the reactivity ratios, respectively, for the monomers I and MA, defined as ${ }^{[11]}$

$$
\begin{aligned}
r_{\mathrm{I}} & =\frac{\text { rate constant for the reaction of }-\mathrm{IO}_{2}^{\cdot}+\mathrm{I}}{\text { rate constant for the reaction of }-\mathrm{IO}_{2}^{\bullet}+\mathrm{MA}} \\
& =\frac{k_{\mathrm{IOI}}}{k_{\mathrm{IOMA}}} \\
r_{\mathrm{M}} & =\frac{\text { rate constant for the reaction of }-\mathrm{MAO}_{2}^{*}+\mathrm{MA}}{\text { rate constant for the reaction of }-\mathrm{MAO}_{2}^{*}+\mathrm{I}} \\
& =\frac{k_{\mathrm{MAOMA}}}{k_{\mathrm{MAOI}}}
\end{aligned}
$$

The Equation (5) for the oxidative copolymerization of two vinyl monomers resembles the Mayo-Lewis equation $^{[22]}$ for copolymerization of binary systems, except for the definition of the reactivity ratio. Thus, the reactivity ratios give the relative reactivity of the peroxide radicals $\left(-\mathrm{IO}_{2}^{*}\right.$ or $\left.-\mathrm{MAO}_{2}^{*}\right)$ to add on to a monomer of the same kind or the other. In the same way, the rate equations can also be written for I-EA and I-BA oxidative copolymerizations.

The reactivity ratios have been determined from Fineman-Ross ${ }^{[23]}$ and Kelen-Tudos ${ }^{[24]}$ plots for the three series of copolyperoxides and their values are given in Table 2 . The reactivity ratios show that the two monomers are randomly placed along the copolymer chain and the copolymers containing appreciable amounts of the acrylate monomers cannot be obtained since $r_{\mathrm{I}} \gg r_{\mathrm{M}}$. The reactivity ratios further indicate the following reactivity sequence: $\mathrm{MA}<\mathrm{EA}<\mathrm{BA}$, towards the peroxy radical - $\mathrm{IO}_{2}^{\circ}$. In indene, the double bond activated by the aromatic ring and the methylene group in the $\alpha$-position relative to the double bond are responsible for the increased ability of indene to interact with oxygen to form polyperoxide. The reactivity of acrylate monomers could be explained on the basis of $\pi$-electron availability. In general conjugation with the electron withdrawing $\mathrm{C}=\mathrm{O}$ group reduces the electron density around the double bond, making it less available for attack by the radicals.

Table 2. Reactivity ratios of the monomers for the oxidative copolymerization of indene with methyl acrylate, ethyl acrylate and butyl acrylate.

\begin{tabular}{llll}
\hline Method & \multicolumn{1}{c}{$r_{\mathrm{I}}$} & \multicolumn{1}{c}{$r_{\mathrm{M}}$} & $r_{\mathrm{I}} / r_{\mathrm{M}}$ \\
\hline \multirow{4}{*}{ Indene/methyl acrylate } & \\
Fineman-Ross & $24.82 \pm 0.05$ & $0.07 \pm 0.04$ & 355 \\
Kelen-Tudos & $25.59 \pm 0.01$ & $0.08 \pm 0.02$ & 320 \\
& \multicolumn{2}{l}{ Indene/ethyl acrylate } \\
Fineman-Ross & $16.23 \pm 0.01 \quad 0.10 \pm 0.05$ & 162 \\
Kelen-Tudos & $16.07 \pm 0.07$ & $0.10 \pm 0.07$ & 161 \\
& \multicolumn{2}{l}{ Indene/butyl acrylate } \\
Fineman-Ross & $28.98 \pm 0.07 \quad 0.27 \pm 0.09$ & 107 \\
Kelen-Tudos & $28.15 \pm 0.09$ & $0.26 \pm 0.10$ & 108 \\
& & & \\
& &
\end{tabular}


In contrast to MA, the increased $+\mathrm{I}$ effect of the ethyl group in EA reduces the electronegativity of the carbonyl carbon, which in turn has a reduced tendency to draw the $\pi$-electron cloud around it. Similarly, BA should be more reactive than the other two acrylates. Both ${ }^{1} \mathrm{H}$ and ${ }^{13} \mathrm{C}$ NMR spectra are complex since during the oxidation of acrylate monomers, the peroxy radical undergoes cleavage reactions preferentially rather than addition reactions with another acrylate molecule ${ }^{[25]}$ The calculation of the reactivity ratios from NMR results is straightforward. However, the assumption that the cleavage products are absent may introduce some error in the calculated values of the reactivity ratios.

\section{Thermal Degradation}

The copolyperoxides were subjected to thermal analysis. According to Mayo mechanism, ${ }^{[26]}$ vinyl polyperoxides generally undergo random thermal scission at the peroxy bond followed by unzipping of the $\beta$-peroxyalkoxy radicals giving carbonyl compounds. A representative DSC thermogram of IMA2 is given in Figure 5. The DSC thermogram of the copolyperoxides shows a highly exothermic degradation. The enthalpy changes for the thermal degradation obtained from the DSC thermogram are presented in Table 3.

The activation energy for the thermal degradation process was determined by the Kissinger's method. ${ }^{[27]}$ The plots of $\ln \left(\phi / T_{\mathrm{m}}^{2}\right)$ against $1 / T_{\mathrm{m}}$, where $\phi$ is the heating rate and $T_{\mathrm{m}}(\mathrm{K})$, the peak temperature obtained from the DSC data are given in Figure 6 for the copolyperoxides IMA2, IEA2 and IBA2. The slope of this plot gives the activation energy $\left(E_{\mathrm{a}}\right)$ for the process of thermal degradation. The $E_{\mathrm{a}}$ values displayed in Table 3 compare well with the dissociation energy of the O-O bond in dialkyl peroxides, ${ }^{[28]}$ PIP, PMAP, IMA2, IEA2 and IBA2. The thermal stability as inferred from their $E_{\mathrm{a}}$ values ${ }^{[29]}$ shows that PIP is more stable than the other polyperoxides and the $E_{\mathrm{a}}$ values of copolyperoxides fall in between that of the two

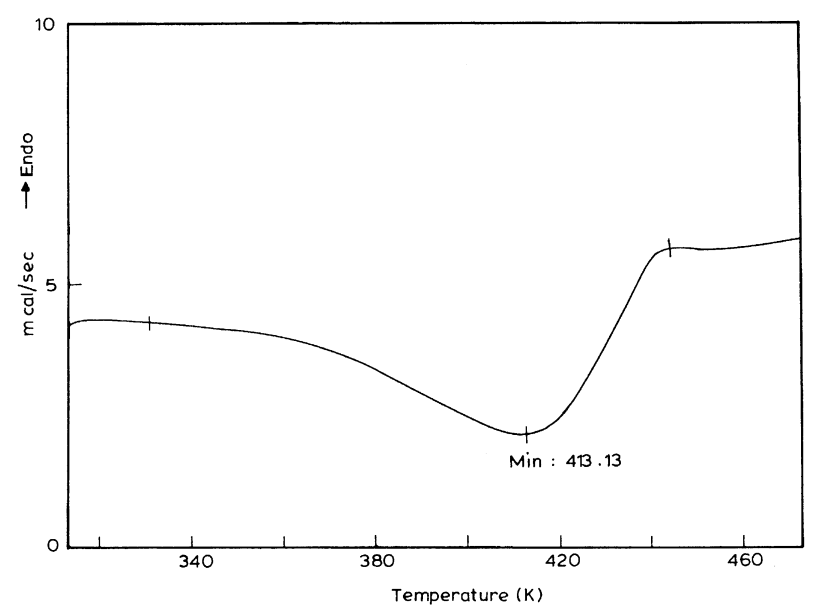

Figure 5. DSC thermogram of IMA2.
Table 3. DSC Data for the polymers investigated.

\begin{tabular}{|c|c|c|c|}
\hline Polymer & $\frac{\Delta H_{\mathrm{d}}^{0}}{\mathrm{cal} \cdot \mathrm{g}^{-1}}$ & $\frac{E_{\mathrm{a}}}{\mathrm{kcal} \cdot \mathrm{mol}^{-1}}$ & $\frac{T_{\mathrm{g}}}{{ }^{\circ} \mathrm{C}}$ \\
\hline \multicolumn{4}{|c|}{ Indene/methyl acrylate } \\
\hline PIP & -292.7 & $47.2 \pm 1.1$ & 39.3 \\
\hline IMA1 & -232.8 & - & 20.0 \\
\hline IMA2 & -264.1 & $37.9 \pm 0.7$ & 2.2 \\
\hline IMA3 & -285.0 & - & -6.6 \\
\hline IMA4 & -223.2 & - & -18.4 \\
\hline PMAP & -210.3 & $33.0 \pm 1.3$ & -29.3 \\
\hline \multicolumn{4}{|c|}{ Indene/ethyl acrylate } \\
\hline IEA1 & -312.0 & - & \\
\hline IEA2 & -289.9 & $41.6 \pm 0.9$ & \\
\hline IEA3 & -234.6 & - & \\
\hline IEA4 & -198.3 & - & \\
\hline \multicolumn{4}{|c|}{ Indene/butyl acrylate } \\
\hline IBA1 & -267.0 & - & \\
\hline IBA2 & -244.3 & $39.6 \pm 1.1$ & \\
\hline IBA3 & -238.2 & - & \\
\hline IBA4 & -201.2 & - & \\
\hline
\end{tabular}

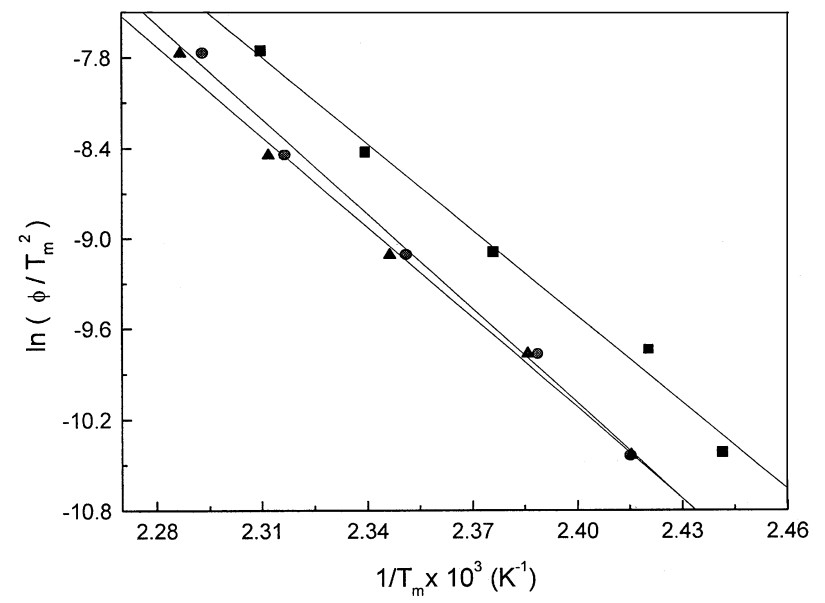

Figure 6. Kissinger's plot of $\ln \phi / T_{\mathrm{m}}^{2}$ against $1 / T_{\mathrm{m}}$ for IMA2 (घ), $\operatorname{IEA} 2(\bullet)$ and IBA2 $(\boldsymbol{\Delta})$.

respective homopolyperoxides. The results reveal that the copolyperoxides undergo a radical chain-scission followed by chain unzipping mechanism during thermal degradation, as reported for other polyperoxides. ${ }^{[30]}$

The copolymer undergoes facile thermal decomposition on heating. The products of decomposition of the copolyperoxides heated in a glass tube at $120^{\circ} \mathrm{C}$ for $2 \mathrm{~h}$ under nitrogen atmosphere were monitored by GPC. It showed that the copolyperoxide degrades to smaller molecular weight products. ${ }^{[31]}$ However, the products of decomposition were not isolated. The thermal degradation in toluene solution of the copolymers IMA2, IEA2 and IBA2 proceed slowly at $75^{\circ} \mathrm{C}$ and the variation in their $\bar{M}_{\mathrm{n}}$ values are shown in Figure 7. The thermal degradation of the polyperoxides depends on the dissociation of the $\mathrm{O}-\mathrm{O}$ bond.

Glass transition temperature $\left(T_{\mathrm{g}}\right)$ for the homo- and copolyperoxides of indene-methyl acrylate measured 


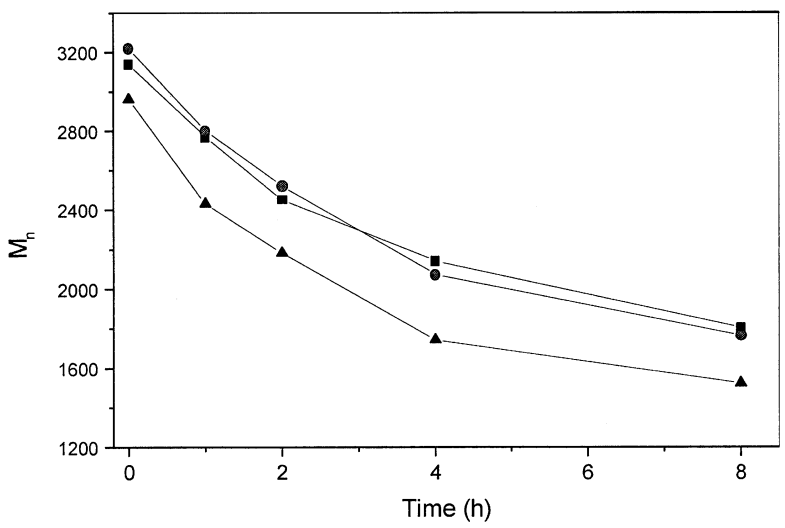

Figure 7. Variations in the molecular weight of the copolyper-

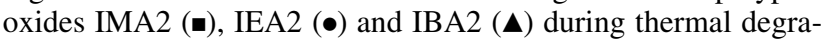
dation at $75^{\circ} \mathrm{C}$ in toluene.

under nitrogen atmosphere at a heating rate $10^{\circ} \mathrm{C} / \mathrm{min}$ are listed in Table 3. The glass transition arises from the onset of backbone motions. The order of mobility can be deduced from the $T_{\mathrm{g}}$ data of the bulk polymers. ${ }^{[32]}$ Higher the $T_{\mathrm{g}}$ value lower would be the flexibility of the polymer. The $T_{\mathrm{g}}$ data indicate PIP to be the most rigid polyperoxide. The $T_{\mathrm{g}}$ values of the copolyperoxides fall in between those of the two respective homopolyperoxides.

\section{Conclusions}

Three series of copolyperoxides of indene with different vinyl monomers have been synthesized and characterized. The compositions of the copolyperoxides and the reactivity ratios of the monomers were obtained from ${ }^{1} \mathrm{H}$ and ${ }^{13} \mathrm{C}$ NMR spectra. The reactivity ratios suggest random placement of the monomers along the copolymer chain and the copolymers containing appreciable amounts of the acrylate monomers cannot be obtained.

The copolyperoxides undergo highly exothermic degradation. The thermal degradation studies of the copolyperoxides support the alternating peroxide units in the copolyperoxide chain. The activation energy for the thermal degradation suggests that the degradation is dependent on the dissociation of the $\mathrm{O}-\mathrm{O}$ bonds of the copolyperoxide chain. Both flexibility and thermal stability decreases with increase in the content of the indene units in the copolyperoxide.

Acknowledgement: We are greatly thankful to Mr. M. Gnana Sekaran (IISc, Bangalore) for recording the NMR spectra and to
Mr. S. Sridhar (CPRI, Bangalore) for providing the DSC facilities.

Received: June 5, 2001

Revised: September 25, 2001

Accepted: October 10, 2001

[1] T. Mukundan, K. Kishore, Prog. Polym. Sci. 1990, 15, 475.

[2] M. M. Mogilevich, Russ. Chem. Rev. 1979, 48, 199.

[3] K. Kishore, T. Mukundan, Nature 1986, 324, 130.

[4] A. K. Nanda, K. Kishore, Macromolecules 2001, 34, 1558.

[5] B. Hazer, A. Kurt, Eur. Polym. J. 1995, 31, 499.

[6] K. Subramanian, K. Kishore, Polymer 1997, 38, 527.

[7] T. Mukundan, V. A. Bhanu, K. Kishore, J. Chem. Soc. Chem. Commun. 1989, 12, 780.

[8] K. Subramanian, K. Kishore, Eur. Polym. J. 1997, 33, 1365.

[9] K. Kishore, K. Ravindran, Macromolecules 1982, 15, 1638.

[10] A. Tager, "Physical Chemistry of Polymers", Mir Publishers 1978.

[11] S. Jayanthi, K. Kishore, Macromolecules 1996, 29, 4846.

[12] F. R. Mayo, A. A. Miller, G. R. Russel, J. Am. Chem. Soc. 1958, 80, 2500.

[13] E. Niki, Y. Kamiya, N. Ohta, Bull. Chem. Soc. Jpn. 1969, 42, 2312.

[14] A. Valvassori, G. Sarton, Adv. Polym. Sci. 1967, 5, 28.

[15] A. K. Nanda, K. Kishore, Macromolecules 2001, 34, 1600.

[16] G. R. Russel, J. Am. Chem. Soc. 1956, 78, 1041.

[17] P. De, D. N. Sathyanarayana, P. Sadasivamurthy, S. Sridhar, Polymer 2001, 42, 8587.

[18] R. E. Cais, F. A. Bovey, Macromolecules 1977, 10, 169.

[19] K. S. Murthy, K. Kishore, V. K. Mohan, Macromolecules 1996, $29,4853$.

[20] F. R. Mayo, A. A. Miller, J. Am. Chem. Soc. 1958, 80 , 2480.

[21] A. H. Fawcett, U. Smyth, Eur. Polym. J. 1989, 25, 791.

[22] F. R. Mayo, F. M. Lewis, J. Am. Chem. Soc. 1944, 66, 1594.

[23] M. Fineman, S. D. Ross, J. Polym. Sci. 1950, 5, 269.

[24] T. Kelen, F. Tudos, J. Macromol. Sci., Chem. 1975, 9, 1.

[25] K. C. Smeltz, E. Dyer, J. Am. Chem. Soc. 1952, 74, 623.

[26] A. A. Miller, F. R. Mayo, J. Am. Chem. Soc. 1956, 78, 1017.

[27] H. E. Kissinger, Anal. Chem. 1957, 29, 1702.

[28] G. Scott, "Atmospheric Oxidants and Antioxidants", Elsevier, London 1965, p. 37.

[29] J. Jayaseharan, A. K. Nanda, K. Kishore, Polymer 2000, 41,5721 .

[30] T. Mukundan, K. Kishore, Macromolecules 1989, 22, 4430.

[31] A. Matsumoto, H. Higashi, Macromolecules 2000, 33, 1651.

[32] F. Heatley, Prog. Nucl. Magn. Reson. Spectrosc. 1979, 13, 47. 